\title{
Experimental tests of nuclear interaction models in few-nucleon systems
}

\author{
E. Stephan*i \\ Institute of Physics, University of Silesia, Katowice, Poland \\ E-mail: elzbieta.stephandus.edu.pl \\ St. Kistryn \\ Institute of Physics, Jagiellonian University, Cracow, Poland \\ E-mail: stanislaw.kistrynduj.edu.pl
}

\section{A. Kozela}

Institute of Nuclear Physics, Polish Academy of Science, Cracow, Poland

E-mail: adam.kozeladifj.edu.pl

Systems of three nucleons can serve as a validation tool for modern approaches to describe the nuclear interaction. At the first stage, investigations were mainly focused on elastic nucleondeuteron scattering, slowly extending to systematic measurements of the deuteron breakup reaction. Intermediate energies, below the threshold for pion production, deserve special attention: it is the region where comparison with exact theoretical calculations is possible, while the sensitivity to various aspects of the interaction, like subtle effects of dynamics beyond the pairwise nucleon-nucleon force (so-called three-nucleon force), Coulomb interaction between protons, or relativistic effects, is significant. Studies of reactions involving 4 nucleons are the next step of complication - they are more sensitive, as expected, to the three-nucleon force. A brief survey of the existing data and upcoming experiments for 3- and 4-nucleon systems at medium energies, with emphasis on the deuteron breakup, is given.

The 8th International Workshop on Chiral Dynamics, CD2015

29 June 2015 - 03 July 2015

Pisa,Italy

\footnotetext{
*Speaker.

${ }^{\dagger}$ Project partially funded by Polish National Science Center from grant DEC-2012/05/B/ST2/02556 and by the European Commission within the Seventh Framework Programme through IA-ENSAR (contract no. RII3-CT-2010262010).
} 


\section{Introduction}

Nuclear interactions can be described in terms of so-called realistic nucleon-nucleon (NN) potentials or within Chiral Perturbation Theory (ChPT), recently undergoing vibrant development. The first approach, very successful in $2 \mathrm{~N}$ systems, when applied to $3 \mathrm{~N}$ systems provides "fairly good" description, but requires consideration of additional dynamics. Such dynamics are related to suppressed internal degrees of freedom and can affect observables in any system beyond $2 \mathrm{~N}$. The Fujita-Miyazawa force [W] is the basic example of such a mechanism, called more generally a threenucleon force (3NF). Similarly, studies of 4N systems would call for additional inclusion of a fournucleon force (4NF), even though it is expected to be a much weaker ingredient of the dynamics. Theoretical calculations comprising precise NN potentials (e.g. CD Bonn []], AV18 [B]], Nijm I and Nijm II [四]) with sophisticated models of 3NF (e.g. TM99 [[]], Urbana IX [目], Illinois [四]) have reached maturity. It is also the case for to the calculations with the explicit $\Delta$ isobar degree of freedom, routinely including the $3 \mathrm{NF}$ [ [8]. These two ways of constructing potentials are taken as a benchmark for $3 \mathrm{~N}$ systems in the medium energy domain. On the other hand, the novel approach of ChPT has many advantages, discussed in detail in a number of theoretical papers (see e.g. [9, [1, प]]). Among others, one should mention preserved symmetries of QCD, systematic treatment of the interaction with naturally appearing 3NF's (and 4NF's etc.) at given order and, last but not least, the possibility to estimate the uncertainty of results.

Regardless of the theoretical approach applied, a data base is needed with scope and precision adequate to validate the predictions. In this paper existing and upcoming data for $3 \mathrm{~N}$ systems at medium energies are discussed. They are compared to theoretical calculations of the first type, since currently the full ChPT calculations are only available up to N2LO (next-to-next-to-leading order), at which the required precision is not reached. There are indications that even N4LO is required to produce sizeable $3 \mathrm{NF}$ contributions, which are necessary to resolve the existing problems [W]. Both, the successes and deficiencies in description of the experimental data provided by "realistic potentials" are challenges for the future ChPT calculations.

\section{Experimental studies of $3 \mathrm{~N}$ systems}

The importance of $3 \mathrm{NF}$ contributions for a proper description of systems of more then two

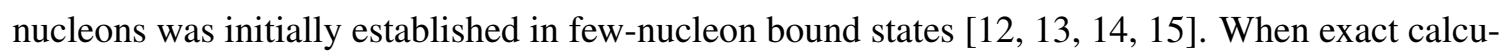
lations including $3 \mathrm{NF}$ became available for observables in the nucleon-deuteron elastic scattering, models of $3 \mathrm{NF}$ turned out to be an efficient remedy for a long persisting problem of describing the cross section for this process [16, [7], [8]. Though at beam energies above $100 \mathrm{MeV}$ per nucleon certain problems with describing the data have remained (cf. [एप] and references therein), improvement in describing the cross section data by including 3NFs is still considerable. On the other hand, precise experimental data demonstrate both successes and difficulties of the current models in describing analyzing powers, spin transfer and spin correlation coefficients. This indicates problems with the spin part of the models of the $3 \mathrm{~N}$ interaction. The current status of experiments searching for $3 \mathrm{NF}$ effects in the elastic scattering is presented in Ref. [20]

In addition to bound states and elastic scattering, the $3 \mathrm{~N}$ system is studied via the nucleondeuteron breakup reaction, which is characterized by variety of kinematic configurations in its 
3-body final state. It opens a way to study kinematic dependencies of $3 \mathrm{NF}$ effects, giving possible insight into the underlying dynamics. Similarly to the elastic scattering, in addition to differential cross section for the breakup reaction, observables related to nuclear polarization are studied, like vector and tensor analyzing powers and higher-rank polarization observables: spin-correlation coefficients and polarization transfer coefficients. For the systematic reviews of the data of the $3 \mathrm{~N}$ breakup see [201, 221, 22]].

The goal of measurements in the domain of few-nucleon systems is to validate the existing calculations or, alternatively, to point out the regions of missing dynamical ingredients. For that purpose high statistical and systematic precision of the collected data is just one basic requirement. To fully describe the transition matrix, a so-called complete set of observables is required - such an ambitious task had been undertaken at RIKEN [23]. On the other hand, even studies of one, or a few observables can lead to important conclusions, provided a significant phase-space region is covered and a set of beam energies is examined in a systematic way. A simple example supporting such a strategy is shown in Fig. $\mathbb{l}$, where the behaviour of the tensor analyzing power of the elastic $d-p$ scattering, $T_{22}^{e l}$, is studied. In order to examine behaviour of $T_{22}^{e l}$ in detail and to compare the data measured at 100,130 and $140 \mathrm{MeV}$, the net effect of 3NF for this observable is studied. The $T_{22}^{e l}$ values obtained with the pure CD Bonn potential $\left(T_{22}^{2 N}\right)$ were subtracted from both the experimental results and the predictions obtained with inclusion of the TM99 $3 \mathrm{NF}\left(T_{22}^{3 N+2 N}\right)$. In this way, the zero level in Fig. $\square$ corresponds to pure NN interaction (in terms of the CD Bonn potential) and any departure from zero can be interpreted as the effect of 3NF. The same procedure was applied for all three beam energies. Below $120^{\circ}$ the predicted effects of the TM99 3NF depend significantly on energy, while at larger angles they are almost independent of the beam energy. Behaviour of the data is very different: below $120^{\circ}$ a majority of the data points is consistent with zero, i.e. they are well described by the pure $\mathrm{NN}$ force predictions. At larger angles, adding $3 \mathrm{NF}$ improves the description significantly in the case of the measurement at $130 \mathrm{MeV}$ beam energy, but for the beam energy of $140 \mathrm{MeV}$ the predicted effect of 3NF is not large enough to reproduce the data, while at $100 \mathrm{MeV}$ the predicted influence of $3 \mathrm{NF}$ is too strong (even though statistical significance of the results at $100 \mathrm{MeV}$ is not high). It is quite clear that limiting the studies to just one of those energies or to a small range of angles could lead to haphazard conclusions.

As far as a systematic approach is considered, studies of the breakup reaction are still far behind the elastic scattering experiments. Yet the situation has been significantly improved by the experimental program carried out at KVI Groningen (The Netherlands). High precision experimental data for cross section, vector (proton) analyzing power and vector and tensor (deuteron) analyzing powers were collected with the use of detection systems covering large part of phase space for the ${ }^{1} \mathrm{H}(\overrightarrow{\mathrm{d}}, \mathrm{pp}) \mathrm{n}$ and ${ }^{2} \mathrm{H}(\overrightarrow{\mathrm{p}}, \mathrm{pp}) \mathrm{n}$ reactions. Usage of multidetector systems, SALAD [R4] and BINA [22] with significant solid angle coverage provided not only very rich data sets but also good opportunities for controlling consistency of the results. Overview of those experiments is given in Table 1. Comparison of the results with the calculations shows a mixed picture, not very different from the one observed for elastic scattering: in the case of the differential cross section the agreement between experiment and theory is improved by including $3 \mathrm{NF}$, while for vector and tensor analysis powers in some cases the $3 \mathrm{NF}$ effects are negligible, and in other cases the inclusion of $3 \mathrm{NF}$ drives the prediction off the data. Part of the collected data is still being analyzed.

In the breakup reaction, in contrast to the elastic scattering, a variety of kinematic config- 


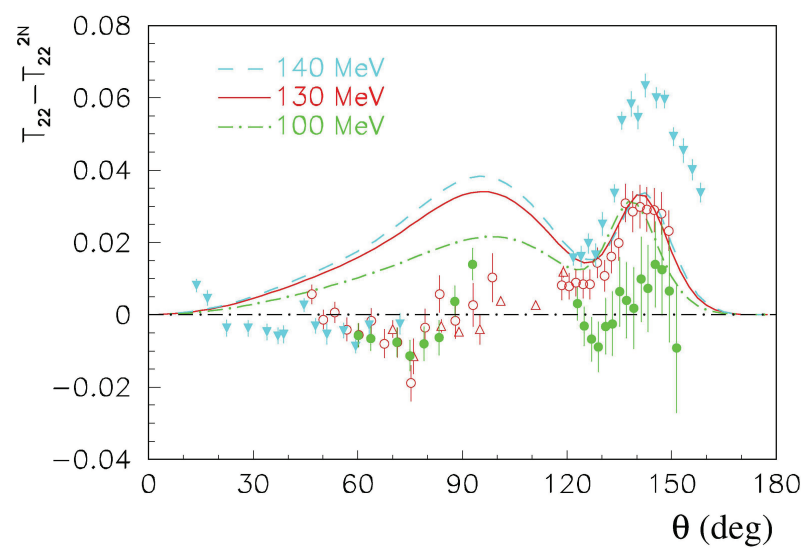

Figure 1: Net effect of $3 \mathrm{NF}$ on $T_{22}^{e l}$ for elastic d-p scattering measured at $100 \mathrm{MeV}$ (full dots), at $130 \mathrm{MeV}$ (open dots - [D25], open triangles - [26] ) and at $140 \mathrm{MeV}$ beam energy (full triangles - [[23]) compared to the predicted effect of the TM99 3NF.

Table 1: Basic parameters of experiments performed at KVI Groningen (The Netherlands) and the Research Center Juelich (Germany).

\begin{tabular}{|l|l|l|l|l|l|l|}
\hline Experiment & $d p 100$ & $d p 130$ & GeWall & $d p 160$ & pd135 & pd190 \\
\hline \hline Beam & $\mathrm{d}$ & $\mathrm{d}$ & $\mathrm{d}$ & $\mathrm{d}$ & $\mathrm{p}$ & $\mathrm{p}$ \\
energy MeV & 100 & 130 & 130 & 160 & 135 & 190 \\
MeV/nucleon & 50 & 65 & 65 & 80 & 135 & 190 \\
\hline Polarization & vector\& tensor & vector\& tensor & vector & - & $\mathrm{p}$ vector & p vector \\
\hline Target & $\mathrm{LH}_{2}$ & $\mathrm{LH}_{2}$ & $\mathrm{LH}_{2}$ & $\mathrm{LH}_{2}$ & $\mathrm{LD}_{2}$ & $\mathrm{LD}_{2}$ \\
\hline Detector & SALAD & BINA & GeWall & BINA & BINA & BINA \\
\hline
\end{tabular}

urations of the final state allows for locally amplified sensitivity to relativistic effects or to the Coulomb force between protons. As soon as the first calculations dealing with the task of including long range (shielded) Coulomb force were performed [ [ $]$, the problem of describing the data measured in configurations close to the so-called Final State Interaction (FSI) was solved [27]. In order to study the Coulomb force effects and their interplay with 3NF, a dedicated measurement has been carried at Forschungszentrum Juelich to investigate the forward region of laboratory angles in the deuteron-proton reactions at $130 \mathrm{MeV}$. A Germanium Wall (GeWall) detector with high angular resolution was used for that purpose. A large influence of Coulomb effects on the differential cross section of the breakup reaction was found [[28].

The next step of complication is to perform studies of reactions involving 4 nucleons, which are expected to be sensitive to subtle dynamics beyond the pairwise nucleon-nucleon interaction. Recently, there is progress in very complex ab-initio calculations for such systems, see e.g. [29]. The data base is, so far, much smaller than for the $3 \mathrm{~N}$ systems and, at medium energies, usually limited to studies of elastic scattering. Therefore two cases of medium energy measurements of the ${ }^{2} \mathrm{H}(\mathrm{d}, \mathrm{dp}) \mathrm{n}$ reaction are worth mentioning, both performed at KVI Groningen with the use of BINA 
detector, at beam energies of $130 \mathrm{MeV}$ [B] $]$ and $160 \mathrm{MeV}$ (data under analysis).

Availability of medium energy beams of the lightest ions is nowadays very limited. It is therefore beneficial for experimental few-nucleon physics that while building the hadron therapy center at Institute of Nuclear Physics, PAN Krakow (Poland), an experimental area for nuclear physics studies was also created. The new facility in Cyclotron Center Bronowice (CCB) provides proton beam accelerated to the energy of $230 \mathrm{MeV}$, which can be reduced by a very precise degrader system continuously down to $70 \mathrm{MeV}$. In order to continue studies of few-body physics, the BINA detector was moved to CCB. Commissioning runs have been performed, so far with solid $\mathrm{CH}_{2}$ and $C D_{2}$ targets. The on-line $\triangle E-E$ spectra obtained in the forward part of the BINA detector (Wall) in coincidence with a particle registered in the central/backward part (Ball) are shown in Fig. \. The chosen angular range of particles in Ball corresponds to deuterons from the elastic $\mathrm{p}$ - $\mathrm{d}$ scattering (in the case of $\mathrm{CH}_{2}$ target) and from the elastic d-d scattering (in the case of $C D_{2}$ target). The observed spectra confirm good separation of deuteron and proton branches and thus prove feasibility of clean identification of the reaction channels.
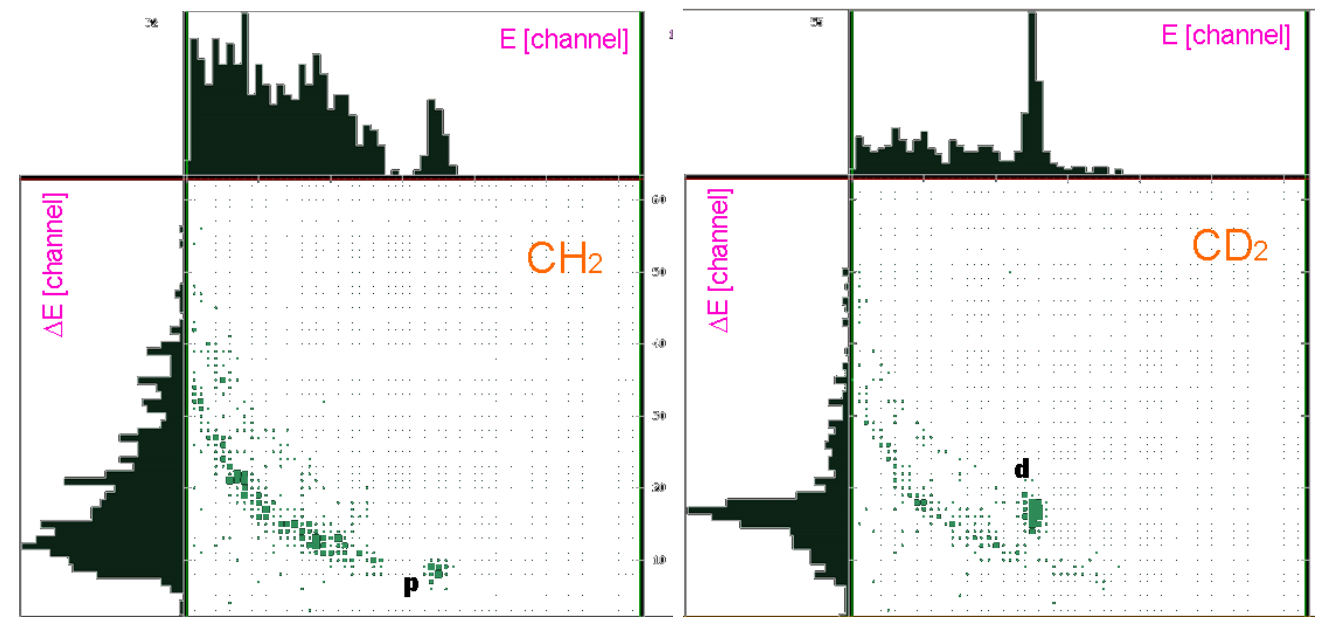

Figure 2: On-line $E-\Delta E$ spectra collected during a test run with the BINA detector installed at CCB Krakow: left panel: spectrum taken with $\mathrm{CH}_{2}$ target, right panel: spectrum taken with $\mathrm{CD}_{2}$ target; spots related to elastically scattered protons (p) and deuterons (d), respectively, are clearly visible; for details see text.

\section{Kinematic variables for description of the breakup reaction}

Each experiment, studying the deuteron breakup reaction by collision with protons in a large part of phase space and with good or moderate angular and energy resolutions, provides very large amount of data - hundreds of data points over 5-dimensional space. This calls for a new method of comparing the data with theory and to express in a systematic way the effects observed at various beam energies.

Kinematics of few-body reactions can be described in many equivalent ways, e.g. in terms either of particle emission angles and energies, or of Jacobi momenta [B]], convenient description of reactions with three (or more) particles in the final state. The number of independent variables depends on the number of particles in the final state (precisely - on the number of components of all 
particle momenta) and on constrains set by momentum and energy conservation laws. Additional constrains, like an axial symmetry of reactions with unpolarized particles, may further reduce that number. A detail discussion of a few-body kinematics can be found in [B2].

In the following, a description in terms of invariants is discussed. Mandelstam variables $s, t$ and $u$ in the case of elastic scattering are defined in an unique way and only two of them are independent. For a reaction with a three-body final state: $a+b \rightarrow 1+2+3$, with final 4-momenta $p_{1}, p_{2}, p_{3}$, more independent invariants can be defined, e.g. several "s-like" variables: $s=\left(p_{a}+p_{b}\right)^{2}$, where $p_{a}, p_{b}$ denote the 4-momenta of particles in the entrance channel (exactly as in the case of the elastic scattering) and, analogously $s_{12}=\left(p_{1}+p_{2}\right)^{2}, s_{23}=\left(p_{2}+p_{3}\right)^{2}$ for the exit channel $\left(s_{13}\right.$ is not independent). Here $s$ in the entrance channel describes the total energy available in the system, while $\sqrt{s_{12}}$ is the invariant mass of the subsystem of particles 1 and 2. Two additional independent variables related to the 4-momentum transfer can be also defined: $t_{a 1}=\left(p_{a}-p_{1}\right)^{2}$ and $t_{b 2}=\left(p_{b}-p_{2}\right)^{2}$

For the breakup reaction, $p+d \rightarrow p^{(1)}+p^{(2)}+n$, in a purely kinematic sense, a deuteron can be described as a proton-neutron pair moving together with a total 4-momentum of $p_{d}$, so roughly each of these particles has a 4-momentum of $p_{d} / 2$. In the exit channel there are two indistinguishable particles and one cannot say which proton, $p^{(1)}$ or $p^{(2)}$, was bound (or free) before the reaction. For all these reasons, we proposed the following set of variables [R2]:

- $s_{p p}$ for a pair of protons in the exit channel,

- $s_{p n} \equiv s_{p^{(1)} n}$ for a proton-neutron pair - arbitrarily the pair with a lower $s_{p n}$ value can be chosen,

- $t_{n}=\left(p_{d} / 2-p_{n}\right)^{2}$, which corresponds to the 4-momentum transfer from a bound neutron in the entrance channel to a free neutron in the exit channel,

- $t_{p}=\left(p_{p}-p_{p}^{(2)}\right)^{2}$, which corresponds to the 4-momentum transfer from an unbound proton to one of the two protons in the exit channel - arbitrarily we choose the proton, which was not used in the calculation of $s_{p n}$.

Use of combinations of invariant variables related to 4-momentum transfers and invariant masses for description of the breakup reaction is not a completely novel technique. However, it was rather applied for specific purposes, not for systematic comparisons of data sets. For example, the so-called Chew-Low Plot was used to identify events originating from quasi-elastic scattering. [B3, [34].

Both, qualitative considerations and precise calculations indicate that the kinetic energy corresponding to the relative motion of the two protons, $E_{r e l}^{p p}$, is an important parameter in studies of Coulomb-force effects. This quantity, equal to the total kinetic energy of the two protons in their center-of-mass reference frame, can be calculated on the basis of the energies and directions of the two protons emitted with momenta $\vec{p}_{1}$ and $\vec{p}_{2}$ as $E_{r e l}^{p p}=\sqrt{\left(E_{1}+E_{2}\right)^{2}-\left(\vec{p}_{1}+\vec{p}_{2}\right)^{2}}-2 m_{p}$.

This variable is, in fact, closely related to $s_{p p}$. One can use the following set of "energy-like" variables:

$$
E_{r e l}^{p p}=\sqrt{s_{p p}}-2 m_{p}
$$




$$
\begin{array}{r}
E_{r e l}^{p n}=\sqrt{s_{p n}}-m_{p}-m_{n}, \\
E_{t r}^{p}=\frac{-t_{p}}{2 m_{p}}, \\
E_{t r}^{n}=\frac{-t_{n}}{2 m_{n}} .
\end{array}
$$

The variables $E_{r e l}^{i j}$ correspond to the kinetic energies of the relative motion of two nucleons ( $i$ and $j$ ), while the variables $E_{t r}^{i}$ - to the energy transfers and, in the case of a nucleon at rest in the entrance channel, to its kinetic energy in the exit channel. Such redefined invariants have more "natural" scale in a direct relation to the beam energy.

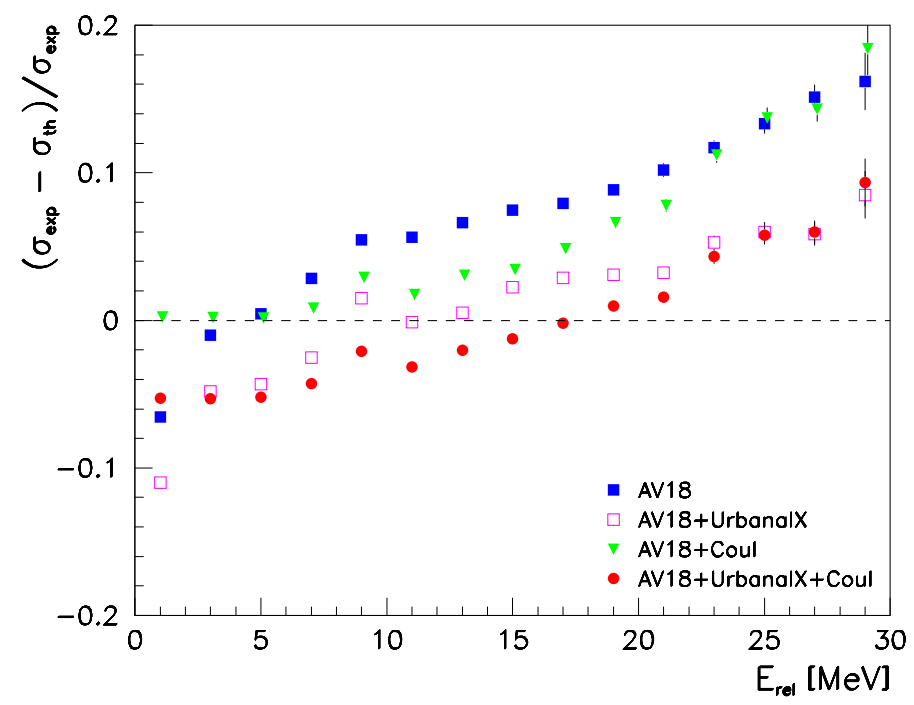

Figure 3: Relative discrepancies between experimental data and theoretical predictions of the breakup cross section as a function of relative energy of the two breakup protons. All calculations use the AV18 NN potential, combined with: Coulomb interaction alone, Urbana IX 3NF alone, and Coulomb interaction with Urbana IX 3NF together [BS], as specified in the panel.

As an example of analysis in invariant coordinates, differential cross section for the ${ }^{1} \mathrm{H}(\overrightarrow{\mathrm{d}}, \mathrm{pp}) \mathrm{n}$ reaction at the beam energy of $130 \mathrm{MeV}$ was studied. The relative differences of the experimental $\left(\sigma_{\text {exp }}\right)$ and theoretical $\left(\sigma_{t h}\right)$ cross section values, $\left(\sigma_{\text {exp }}-\sigma_{t h}\right) / \sigma_{\text {exp }}$, were determined and plotted as a function of $E_{r e l}^{p p}$, see Fig. [1. Theoretical calculations were performed with the use of AV18 potential alone, or with addition of the Urbana IX 3NF or/and Coulomb interaction [35]. As expected, the strongest influence of Coulomb interaction can be observed at the smallest values of $E_{r e l}^{p p}$. In this region the calculations including the Coulomb force are closer to the data than the ones performed without that ingredient. The same conclusion follows from the similar comparison performed on the basis of the coupled channel calculations with an explicit $\Delta$, with and without Coulomb interaction, see [27]. On the other hand, $3 \mathrm{NF}$ effects play a significant role in a wider region of $E_{r e l}^{p p}$, up to its highest values. The calculations including both ingredients provide the best description of the data, if the whole range of $E_{r e l}^{p p}$ is taken into account. The remaining small discrepancies $( \pm 5 \%)$ can be attributed either to systematic uncertainties of the data, or to contribution of relativistic effects. 


\section{Summary and Outlook}

All the dynamical effects studied in $3 \mathrm{~N}$ systems vary with energy and appear with different strength in certain observables and phase space regions. This calls for systematic investigations of a possibly rich set of observables determined in a wide range of energies. The data base for the breakup reaction is rich in data, but not yet systematic in energy. The region of energies below, but not far from pion production threshold, is interesting for testing calculations performed in a relativistic regime [36, 37]]. For that purpose the experiment with the use of WASA detector at the COSY cyclotron (Forchungszentrum Juelich, Germany) was performed for three deuteron beam energies: 340, 380 and $400 \mathrm{MeV}$ (170, 190 and $200 \mathrm{MeV} /$ nucleon). The other "gap" in the energies studied so far, roughly between 100 and $160 \mathrm{MeV} /$ nucleon, will be investigated with BINA at CCB.

Kinematic configurations of the proton-induced deuteron breakup reaction can be defined in terms of invariant coordinates, a convenient way to analyse the influence of $3 \mathrm{NF}$, Coulomb or relativistic effects. The studies can be carried out in a function of beam energy and provide cross checks with the elastic scattering. Such investigations are currently under way on the basis of existing data and theoretical calculations. Up to now, they confirm importance of the Coulomb interaction for correct description of the reaction, in particular at low relative energies of two protons, but extending over wide phase space regions.

The existing data for cross sections support the predicted importance of three-nucleon force in descriptions of $3 \mathrm{~N}$ systems at medium energies. The most important questions concern polarization observables, especially tensor analyzing powers. It will be very interesting to confront the data with the calculations employing potentials derived from ChPT.

\section{References}

[1] J. Fujita and H. Miyazawa, Prog. Theor. Phys. 17 (1957) 360.

[2] R. Machleidt, F. Sammarruca and Y. Song Phys. Rev. C 53 (1996) R1483;

R. Machleidt Phys. Rev. C 63 (2001) 024001.

[3] R.B. Wiringa, V.G.J. Stoks and R. Schiavilla Phys. Rev. C 51 ( 1995$) 38$.

[4] V.G.J. Stoks, R.A.M. Klomp, C.P.F. Terheggen and J.J. de Swart Phys. Rev. C 49 (1994) 2950.

[5] S. Coon and H.K. Han Few-Body Syst. 30 (2001) 131.

[6] B.S. Pudliner, V.R.Pandharipande, J. Carlson, S.C. Pieper and R.B. Wiringa Phys. Rev. C 56 (1997) 1720 .

[7] S.C. Pieper, V.R. Pandharipande, R.B. Wiringa and J. Carlson Phys. Rev. C 64 (2001) 014001.

[8] A. Deltuva, A.C. Fonseca and P.U. Sauer Phys. Rev. C 72 (2005) 054004;

A. Deltuva, A.C. Fonseca and P.U. Sauer Phys. Rev. C 73 (2005) 057001.

[9] P.F. Bedaque and U. van Kolck Annu.Rev.Nucl.Part.Sci. 52 (2002) 339.

[10] E. Epelbaum, H.W. Hammer and U. Meissner Rev. Mod. Phys. 81 (2009) 1773.

[11] R.Machleidt and D.R.Entem, Phys. Rep. 503 (2011) 1.

[12] M. Viviani Nucl. Phys. A 631 (1998) 111c.

[13] A. Nogga, H. Kamada and W. Glockle Phys. Rev. Lett. 85 (2000) 944. 
[14] S.C. Pieper and R.B. Wiringa Ann. Rev. Nucl. Part. Sci. 51 (2001) 53.

[15] P. Navratil, V.G. Gueorguiev, J.P. Vary, W.E. Ormand and A. Nogga Phys. Rev. Lett. 99 (2007) 042501.

[16] H. Witala, W. Glockle, D. Huber, J. Golak and H. Kamada Phys. Rev. Lett. 81 (1998) 1183.

[17] K. Hatanaka et al. Phys. Rev. C 66 (2002) 044002.

[18] P. Mermod et al. Phys. Lett. B 597 (2004) 243.

[19] A. Ramazani-Moghaddam-Arani et al. Phys. Rev. C 78 (2008) 014006.

[20] N. Kalantar-Nayestanaki, E. Epelbaum, J.G. Meschendorp and A. Nogga Rep. Prog. Phys. 75 (2012) 016301.

[21] K. Sagara Few Body Syst. 48 (2010) 59.

[22] St. Kistryn and E. Stephan Jour. Phys. G 40 (2013) 063101.

[23] K. Sekiguchi et al. Phys. Rev. C 70 (2004) 014001.

[24] N. Kalantar-Nayestanaki et al. Nucl.Instr.Meth. A 444 (2000) 591.

[25] E. Stephan et al. Phys. Rev. C 76 (2007) 057001.

[26] H. Mardanpour et al. Eur. Phys. J. 31 (2007) 383.

[27] St. Kistryn et al. 2006 Phys. Lett. B 64123.

[28] I. Ciepal et al. Few. Body Sys. A 56 (2015) 665.

[29] A. Deltuva Few. Body Sys. A 55 (2013) 621.

[30] A. Ramazani-Moghaddam-Arani et al. Phys.Lett.B 725 (2013) 282.

[31] W. Gloeckle The Quantum Mechanical Few-Body Problem, Springer Verlag Berlin 1983.

[32] N. Byckling and K. Kajantie Particle Kinematics, Wiley, New York 1973.

[33] G. Baur Z. Physik A 277 (1976) 147.

[34] J. Stepaniak Acta Phys. Pol. B 27 (1996) 2971.

[35] A. Deltuva Phys. Rev. C 80 (2009) 064002.

[36] H. Witala, J. Golak and R. Skibinski Phys. Lett. B 634 (2006) 374;

R. Skibinski, H. Witala and J. Golak Eur. Phys. J. A 30 (2006) 369.

[37] H. Witala et al. Phys. Rev. C 83 (2011) 044001. 